\title{
Improving Gender Classification Accuracy in the Wild
}

\author{
Modesto Castrillón-Santana ${ }^{\star}$, Javier Lorenzo-Navarro, \\ and Enrique Ramón-Balmaseda \\ SIANI \\ Universidad de Las Palmas de Gran Canaria, Spain \\ mcastrillon@siani.es
}

\begin{abstract}
In this paper, we focus on gender recognition in challenging large scale scenarios. Firstly, we review the literature results achieved for the problem in large datasets, and select the currently hardest dataset: The Images of Groups. Secondly, we study the extraction of features from the face and its local context to improve the recognition accuracy. Different descriptors, resolutions and classifiers are studied, overcoming previous literature results, reaching an accuracy of $89.8 \%$.
\end{abstract}

Keywords: gender recognition, local context, head and shoulders, LBP, HOG, in the wild.

\section{Introduction}

Gender is a valid demographic characteristic for different applications that has recently attracted commercial attention in the context of audience analysis and advertisement.

Different approaches have tackled the problem of automatic gender recognition. Most recent works have basically considered the face pattern to solve the problem [2]314]. Other approaches have made use of non facial features such as the whole body, the hair or clothing [4]13. However, those approaches including non facial features, have rarely considered uncontrolled large datasets, i.e. the gender recognition in the wild. In this context, the evaluation must tackle more variability in terms of 1) identities, aging and ethnicity, 2) pose and illumination control, and 3) low resolution images.

The contributions of this paper rely firstly on the addition to the information provided by the face, of features extracted from the head local context. Those features are studied at different resolutions, and their possibilities analyzed as additional features for the problem. Another main element of this paper is the use of large databases that are closer to real gender classification scenarios than those small databases obtained in controlled environments.

\footnotetext{
* This work was partially funded by the Institute of Intelligent Systems and Numerical Applications in Engineering (SIANI) and the Computer Science Department at ULPGC.
} 


\subsection{Previous Work in Large Datasets}

Table 1. Gender recognition accuracy in the previous literature. Refer to each reference for experimental setup details.

\begin{tabular}{|c|c|c|c|}
\hline Reference & Dataset & Protocol & Accuracy \\
\hline$[19$ & LFW & Subset $7443 / 13233$ & $94.81 \%$ \\
{$[20$} & LFW & Subset $7443 / 13233$ & $98.01 \%$ \\
\hline 7 & LFW & BEFIT protocol & $97.23 \%$ \\
\hline 7 & GROUPS & Subset $15579 / 28231$ & $84.55-86.61 \%$ \\
{$[12$} & GROUPS & Subset 22778/28231 & $86.4 \%$ \\
\hline $\mathbf{5}$ & MORPH & Subset & $88 \%$ \\
{$[17$} & MORPH & Subset & $97.1 \%$ \\
\hline
\end{tabular}

We argue that small databases are not representative for a real world scenario where a gender recognizer must cope with thousands of people, like for example a mall scenario. For that reason, we have reviewed the literature to detect state-ofthe-art accuracies obtained for large public databases that contain many different identities acquired without controlled conditions. As far as we know, Table 1 presents the best accuracies reported on large datasets in the recent literature. The datasets studied are The Image of Groups (GROUPS) [10, Labeled Faces in the Wild (LFW) [1], and MORPH [18].

Observing in detail Table 11 there is not much space for improvement in datasets such as LFW and MORPH. Certainly, both datasets present a set of characteristics that might affect the impressive resulting performance. Indeed, in both datasets the same identity includes multiple samples. Additionally, sample images of both genders are not equally represented in the set, i.e. the number of samples corresponding to the male class is significantly larger. On the other side, the GROUPS dataset presents unrestricted imagery with balanced presence of both classes, reporting the lowest accuracy in the recent works. For all those reasons, we have selected to focus on the GROUPS dataset, that represents, in our opinion, the wildest available dataset for the problem, see Figure 1a.

\section{Representation and Classification}

Local descriptors have recently attracted the attention of researchers involved in the facial analysis community [21]. We will focus particularly on Local Binary Patterns [16] (LBPs) and Histograms of Oriented Gradients [8]. Both descriptors have already been used successfully for facial analysis [915.

Facial analysis with LBP is currently adopted considering a concatenation of histograms of a predefined grid. This approach was adopted for LBP by Ahonen et al. [1]. According to that work the face is divided into regions where the LBP operator is computed and later their corresponding histograms concatenated, following a Bag of Words scheme [6, into a single histogram. On the other side, HOG encloses a histogram in its definition. The pattern is scaled to a normalized resolution, and later a grid is defined. 


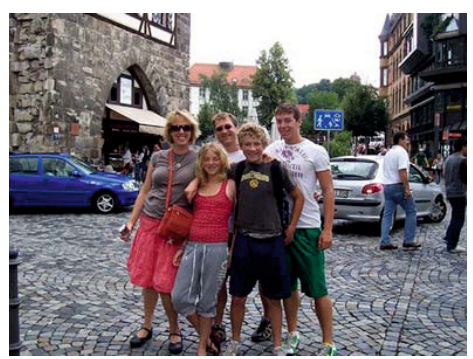

(a)

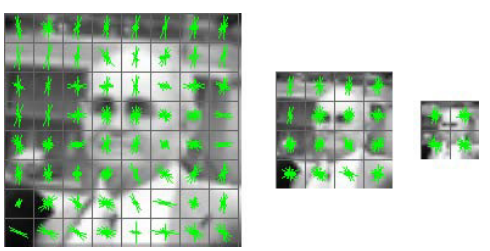

(b)

Fig. 1. a) A GROUPS sample. b) Relative size of the different patterns used including the local context: respectively $64 \times 64,32 \times 32$ and $16 \times 16$ faces with head and shoulders context. Their respective HOG grid computed is also depicted.

For classification purposes, we will compare two different approaches. The first one will study the addition of features to an initial feature vector filled exclusively with features extracted from the face. In this scenario, two well known classifiers are compared: SVM with linear kernel, and bagging making use of SVM classifiers based on linear kernels.

In the second scenario, instead of combining features of different nature in the feature vector, we focus on the combination of the outputs provided by the different classifiers in a first stage. Their respective scores are combined in a second classification stage. This combination is compared based on different known classification techniques such as: SVM (linear kernel), bagging, naive Bayes, Nearest Neighbor (NN) and C4.5.

\section{Results}

In the experimental setup, we have adopted a k-fold cross-validation, partitioning the dataset into $k$ subsets, repeating $k$ times the experiment using a subset to test the model with the other $k-1$ subsets. In order to be comparable to previous works, we made use of the 5 -folds defined in the work by Dago et al. [7.

The Uniform LBP descriptor is used only for the face area, at two different resolutions: $59 \times 65$ and $100 \times 110$, defining a $5 \times 5$ grid. When using HOG as descriptor, the face area is used just with the $59 \times 65$ resolution, but the head and shoulders pattern was tested at different resolutions: $16 \times 16,32 \times 32$ and $64 \times 64$, see Figure 1 b. On each resolution the cell contains $8 \times 8$ pixels, each block $2 \times 2$ cells, the histogram contains 9 bins, and L2-hys as norm for the normalization stage 8 .

\subsection{Extending the Feature Vector}

Firstly, we performed a comparison using just the facial information, i.e. the inner face details (Face), and its local context defined by the head and shoulders 
area (HS). Tables 2 and 3 present respectively those results. The face pattern resolution used in Table 2 was $59 \times 65$ pixels, with an inter-eye distance of 26 pixels. For comparison with a baseline, we have also included the results achieved with a classifier trained with the first 100 PCA components.

Table 2. Gender recognition accuracy (in brackets results per class: female/male) achieved using PCA, HOG or Uniform LBP features extracted from the face pattern. The table reports the results achieved using the 5-fold cross correlation experiment defined by Dago et al. SVM linear and Bagging are used for classification.

\begin{tabular}{|c|c|c|c|c|}
\hline \multirow{2}{*}{$\begin{array}{c}\text { Pattern and } \\
\text { features }\end{array}$} & \multicolumn{3}{|c|}{ Test set GROUPS-Dago 5-folds subset } \\
Acc. linear & AUC & \multicolumn{2}{|c|}{ Bagging } \\
Face PCA & 0.773 & 0.773 & 0.7749 & 0.801 \\
& $(0.773 / 0.774)$ & & $(0.779 / 0.770)$ & \\
\hline Face HOG & 0.801 & 0.801 & 0.822 & 0.898 \\
& $(0.797 / 0.805)$ & & $(0.84 / 0.800)$ & \\
\hline \multirow{2}{*}{ Face LBP } & $\mathbf{0 . 8 3 8}$ & 0.838 & $\mathbf{0 . 8 3 8}$ & 0.910 \\
& $(0.842 / 0.834)$ & & $(0.863 / 0.814)$ & \\
\hline
\end{tabular}

Table 3 presents the results using information extracted from the face and its local context. The head and shoulders were analyzed at different resolutions: $16 \times 16,32 \times 32$ and $64 \times 64$, with their respective inter-eye distances of $2.5,5$ and 10 pixels. Observe, that the facial resolution contained in the head and shoulders pattern is lower up to ten times compared to the results reported in Table 2. Even though, the best accuracy is rather similar, almost $84 \%$ using the $64 \times 64$ head and shoulders pattern, than exclusively the facial pattern at larger resolution. Even considering the smallest pattern, with an inter-eye distance under 3 pixels, the accuracy reaches $66 \%$. That is not a bad result for low resolution images.

Table 3. Gender recognition accuracy (in brackets results per class: female/male) achieved using HOG features extracted from the head and shoulders (HS) pattern using different image dimensions. The table reports the results achieved using the 5fold cross correlation experiment defined by Dago et al. SVM linear and Bagging are used for classification.

\begin{tabular}{|c|c|c|c|c|}
\hline \multirow{2}{*}{$\begin{array}{c}\text { Pattern and } \\
\text { features }\end{array}$} & \multicolumn{4}{|c|}{ Test set GROUPS-Dago 5-folds subset } \\
SVM linear & Acc. & AUC & Bcc. & AUC \\
\hline$H S_{16 \times 16}$ HOG & 0.6608 & 0.661 & 0.659 & 0.687 \\
& $(0.6538 / 0.6684)$ & & $(0.6616 / 0.6564)$ & \\
\hline$H S_{32 \times 32}$ HOG & 0.812 & 0.812 & 0.8099 & 0.865 \\
& $(0.8024 / 0.8216)$ & & $(0.8122 / 0.8076)$ & \\
\cline { 2 - 4 }$H S_{64 \times 64}$ HOG & 0.8298 & 0.829 & $\mathbf{0 . 8 3 9 7}$ & 0.909 \\
& $(0.829 / 0.83)$ & & $(0.8562 / 0.8232)$ & \\
\hline
\end{tabular}


On a second step, we have considered to fuse in a single feature vector, features extracted from different cues. Table 4 presents results combining Uniform LBP or HOG features extracted from the face pattern, with HOG features extracted from the head and shoulders pattern at different resolutions. Bagging reports better accuracy for the experimental setup, while the use of Uniform LBP features seems to work slightly better than HOG. The notorious increase in the face pattern resolution, does not suggest a large improvement in accuracy. The best reported accuracy reaches $88.1 \%$, four points better than our previous results, and $2 \%$ better than the literature for the same dataset, see Table1. These results suggest the importance of the information contained in the facial local context.

Table 4. Gender recognition accuracy (in brackets results per class: female/male) achieved using different representation alternatives. The table reports the results achieved using the 5 -fold cross correlation experiment defined by Dago et al. SVM linear and Bagging are used for classification.

\begin{tabular}{|c|c|c|c|c|c|c|c|c|}
\hline \multirow{4}{*}{$\begin{array}{l}\text { Pattern and } \\
\text { features }\end{array}$} & \multicolumn{8}{|c|}{ Test set GROUPS-Dago 5-folds subset } \\
\hline & \multicolumn{4}{|c|}{ SVM linear } & \multicolumn{4}{|c|}{ Bagging } \\
\hline & \multicolumn{2}{|c|}{ face $59 \times 65$} & \multicolumn{2}{|c|}{ face $100 \times 110$} & \multicolumn{2}{|c|}{ face $59 \times 65$} & \multicolumn{2}{|c|}{ face $100 \times 110$} \\
\hline & Acc. & AUC & Acc. & $\mathrm{AUC}$ & Acc. & AUC & Acc. & AUC \\
\hline Face $\mathrm{HOG}$ & $\begin{array}{c}0.801 \\
(0.797 / 0.805)\end{array}$ & 0.801 & - & - & $\begin{array}{c}0.822 \\
(0.84 / 0.800)\end{array}$ & 0.898 & - & - \\
\hline Face LBP & $\begin{array}{c}0.827 \\
(0.835 / 0.814)\end{array}$ & 0.827 & $\begin{array}{c}0.836 \\
(0.836 / 0.836)\end{array}$ & 0.836 & $\begin{array}{c}0.823 \\
(0.856 / 0.804)\end{array}$ & 0.905 & $\begin{array}{c}0.84 \\
(0.862 / 0.817) \\
\end{array}$ & 0.909 \\
\hline $\begin{array}{l}\text { Face } \mathrm{HOG} \text { and } \\
H S_{16 \times 16} \mathrm{HOG}\end{array}$ & $\begin{array}{c}0.827 \\
(0.828 / 0.827)\end{array}$ & 0.827 & - & - & $\begin{array}{c}0.829 \\
(0.848 / 0.809)\end{array}$ & 0.904 & - & - \\
\hline $\begin{array}{l}\text { Face HOG and } \\
H S_{32} \times 32 \text { HOG }\end{array}$ & $\begin{array}{c}0.852 \\
(0.855 / 0.85)\end{array}$ & 0.852 & - & - & $\begin{array}{c}0.862 \\
(0.881 / 0.843)\end{array}$ & 0.93 & - & - \\
\hline $\begin{array}{l}\text { Face } \mathrm{HOG} \text { and } \\
H S_{64} \times 64 \mathrm{HOG}\end{array}$ & $\begin{array}{c}0.845 \\
(0.851 / 0.84)\end{array}$ & 0.845 & - & - & $\begin{array}{c}0.875 \\
(0.893 / 0.858)\end{array}$ & 0.941 & - & - \\
\hline $\begin{array}{l}\text { Face LBP and } \\
H S_{16 \times 16} \text { HOG }\end{array}$ & $\begin{array}{c}0.838 \\
(0.842 / 0.834)\end{array}$ & 0.838 & $\begin{array}{c}0.844 \\
(0.843 / 0.846)\end{array}$ & 0.844 & $\begin{array}{c}0.838 \\
(0.863 / 0.814)\end{array}$ & 0.910 & $\begin{array}{c}0.845 \\
(0.864 / 0.826)\end{array}$ & 0.915 \\
\hline $\begin{array}{l}\text { Face LBP and } \\
H S_{32} \times 32 \text { HOG }\end{array}$ & $\begin{array}{c}0.859 \\
(0.86 / 0.857)\end{array}$ & 0.859 & $\begin{array}{c}0.862 \\
(0.861 / 0.863)\end{array}$ & 0862 & $\begin{array}{c}0.867 \\
(0.889 / 0.845)\end{array}$ & 0.933 & $\begin{array}{c}0.869 \\
(0.864 / 0.826) \\
\end{array}$ & 0.937 \\
\hline $\begin{array}{l}\text { Face LBP and } \\
H S_{64 \times 64} \mathrm{HOG}\end{array}$ & $\begin{array}{c}0.851 \\
(0.851 / 0.85)\end{array}$ & 0.851 & $\begin{array}{c}0.861 \\
(0.859 / 0.864)\end{array}$ & 0.861 & $\begin{array}{c}0.879 \\
(0.897 / 0.862)\end{array}$ & 0.944 & \begin{tabular}{c|}
$\mathbf{0 . 8 8 1}$ \\
$(0.897 / 0.866)$ \\
\end{tabular} & 0.946 \\
\hline
\end{tabular}

\subsection{Stacking Classifiers}

We went further, and considered an alternative to the inclusion of more features in the feature vector. Instead, we considered a stacking of classifiers in two stages. The first stage is composed by the individual 11 feature vectors described in Tables 3 and 4, and summarized in the following list:

- HOG of the $64 \times 64$ head and shoulders pattern (HSHOG64).

- HOG of the $32 \times 32$ head and shoulders pattern (HSHOG32).

- HOG of the $16 \times 16$ head and shoulders pattern (HSHOG16).

- HOG of the $59 \times 65$ facial pattern $($ FHOG).

- Concatenated LBP histogram extracted from the $59 \times 65$ facial pattern (FLBP). 
- HOG of the $64 \times 64$ head and shoulders pattern, and HOG of the $59 \times 65$ face pattern (HSHOG64-FHOG).

- HOG of the $32 \times 32$ head and shoulders pattern, and HOG of the $59 \times 65$ face pattern (HSHOG32-FHOG).

- HOG of the $16 \times 16$ head and shoulders pattern, and HOG of the $59 \times 65$ face pattern (HSHOG16-FHOG).

- HOG of the $64 \times 64$ head and shoulders pattern, and concatenated LBP histogram of the $59 \times 65$ face pattern (HSHOG64-FLBP).

- HOG of the $32 \times 32$ head and shoulders pattern, and concatenated LBP histogram of the $59 \times 65$ face pattern (HSHOG32-FLBP).

- HOG of the $16 \times 16$ head and shoulders pattern, and concatenated LBP histogram of the $59 \times 65$ face pattern (HSHOG16-FLBP).

Each of the first stage classifier is trained using a SVM with a liner kernel. In the second stage of the stacking classifier, their respective scores are feed into a classifier that is in charge of taking the final decision. For this second stage, we have analyzed the accuracy reported for SVM (linear kernel), Bagging, Naive Bayes, Nearest Neighbor (NN) and C4.5. The results achieved are reported in Table 5. They suggest an improvement, reaching with Naive Bayes almost $90 \%$. The reader may observe, that this accuracy was achieved without using the classifiers based on the largest face pattern, i.e. an inter-eye distance of 26 pixels. Compared to Table 4 for similar facial resolution the improvement is almost $2 \%$. Compared to the literature, see Table 1, the improvement is close to $4 \%$ even using a facial pattern that is twice smaller. The benefits introduced by the descriptors and the face local context are evident.

We have additionally performed a feature selection to reduce the system complexity avoiding the computation of all the classifiers present in the stacking first stage. After sorting attending to the information gain, the resulting accuracy considering as variable the number of classifiers included in the stacking is presented in Figure 2, With just 4 classifiers in the first stage, the system performance achieves an accuracy of $89 \%$ beating those results reported in the previous section and the literature. Those classifiers are: HSHOG64-FLBP, HSHOG32FLBP, HSHOG16-FLBP and HSHOG32-FHOG.

Table 5. Gender recognition accuracy achieved using classifiers stacking. The table reports the results achieved using the 5 -fold cross correlation experiment defined by Dago et al.

\begin{tabular}{|c|c|}
\hline Classifier & Accuracy \\
\hline Naive Bayes & $\mathbf{0 . 8 9 7 8}$ \\
\hline SVM & 0.8736 \\
\hline C4.5 & 0.8336 \\
\hline NN & 0.8662 \\
\hline
\end{tabular}




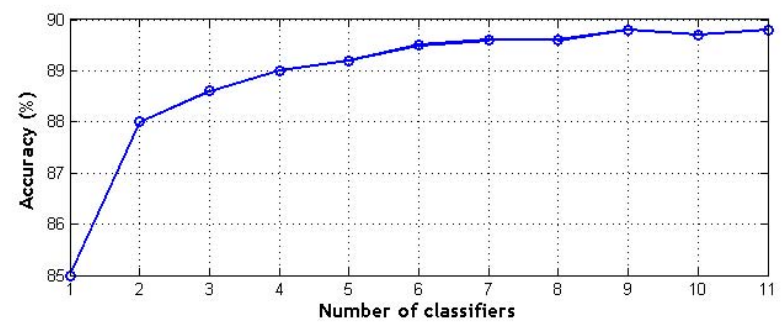

Fig. 2. Accuracy achieved adding more classifiers to the stacking

\section{Conclusions}

In this paper, we have studied gender recognition in large uncontrolled datasets. For that purpose, we have made use of facial and non facial features, in the large database that is currently reporting the lowest accuracy in the literature: The Images of Groups.

The addition of external facial features seem to bring benefits at lower resolution, and the combination with facial features reported better accuracies that the previous literature.

We have used features based on the Uniform LBP and HOG operators, both used widely in similar problems. For classification we have considered the used combination of features in a large dataset, and the stacking of classifiers, each one focused in a particular family of features. The stacking results are particularly better than those obtained when the feature vector is increased, reaching almost $90 \%$. This accuracy is notoriously better than those previously reported in the literature, even if the face pattern considered makes use of a facial resolution at least twice smaller.

In summary, the performance exhibited at lower resolution, is best suited for real scenarios. However, the achieved at high resolution beats state of the art results.

\section{References}

1. Ahonen, T., Hadid, A., Pietikäinen, M.: Face description with local binary patterns: Application to face recognition. IEEE Transactions on Pattern Analysis and Machine Intelligence 28(12) (December 2006)

2. Alexandre, L.A.: Gender recognition: A multiscale decision fusion approach. Pattern Recognition Letters 31(11), 1422-1427 (2010)

3. Bekios-Calfa, J., Buenaposada, J.M., Baumela, L.: Revisiting linear discriminant techniques in gender recognition. IEEE Transactions on Pattern Analysis and Machine Intelligence 33(4), 858-864 (2011)

4. Bourdev, L., Maji, S., Malik, J.: Describing people: A poselet-based approach to attribute classification. In: International Conference on Computer Vision (2011) 
5. Chu, W.-S., Huang, C.-R., Chen, C.-S.: Identifying gender from unaligned facial images by set classification. In: International Conference on Pattern Recognition (ICPR), Istanbul, Turkey (2010)

6. Csurka, G., Dance, C.R., Fan, L., Willamowski, J., Bray, C.: Visual categorization with bags of keypoints. In: Workshop on Statistical Learning in Computer Vision, ECCV, pp. 1-22 (2004)

7. Dago-Casas, P., González-Jiménez, D., Long-Yu, L., Alba-Castro, J.L.: Single- and cross- database benchmarks for gender classification under unconstrained settings. In: Proc. First IEEE International Workshop on Benchmarking Facial Image Analysis Technologies (2011)

8. Dalal, N., Triggs, B.: Histograms of oriented gradients for human detection. In: Schmid, C., Soatto, S., Tomasi, C. (eds.) International Conference on Computer Vision \& Pattern Recognition, vol. 2, pp. 886-893. INRIA Rhône-Alpes, ZIRST655, av. de l'Europe, Montbonnot-38334 (June 2005)

9. Déniz, O., Bueno, G., Salido, J., De La Torre, F.: Face recognition using histograms of oriented gradients. Pattern Recognition Letters 32(12), 1598-1603 (2011)

10. Gallagher, A., Chen, T.: Understanding images of groups of people. In: Proc. CVPR (2009)

11. Huang, G.B., Ramesh, M., Berg, T., Learned-Miller, E.: Labeled faces in the wild: A database for studying face recognition in unconstrained environments. Technical Report 07-49, Univ, of Massachusetts, Amherst (October 2007)

12. Kumar, N., Berg, A.C., Belhumeur, P.N., Nayar, S.K.: Describable visual attributes for face verification and image search. IEEE Transactions on Pattern Analysis and Machine Intelligence (PAMI) (October 2011)

13. Li, B., Lian, X.-C., Lu, B.-L.: Gender classification by combining clothing, hair and facial component classifiers. Neurocomputing 76(1), 18-27 (2012)

14. Mäkinen, E., Raisamo, R.: Evaluation of gender classification methods with automatically detected and aligned faces. IEEE Transactions on Pattern Analysis and Machine Intelligence 30(3), 541-547 (2008)

15. Marcel, S., Rodríguez, Y., Heusch, G.: On the recent use of local binary patterns for face authentication. International Journal of Image and Video Preprocessing, Special Issue on Facial Image Processing (2007)

16. Ojala, T., Pietikäinen, M., Mäenpää, T.: Multiresolution gray-scale and rotation invariant texture classification with local binary patterns. IEEE Trans. on Pattern Analysis and Machine Intelligence 24(7), 971-987 (2002)

17. Ramón-Balmaseda, E., Lorenzo-Navarro, J., Castrillón-Santana, M.: Gender classification in large databases. In: Alvarez, L., Mejail, M., Gomez, L., Jacobo, J. (eds.) CIARP 2012. LNCS, vol. 7441, pp. 74-81. Springer, Heidelberg (2012)

18. Ricanek Jr., K., Tesafaye, T.: MORPH: A longitudinal image database of normal adult age-progression. In: IEEE 7th International Conference on Automatic Face and Gesture Recognition, Southampton, UK, pp. 341-345 (April 2006)

19. Shan, C.: Learning local binary patterns for gender classification on realworld face images. Pattern Recognition Letters 33, 431-437 (2012)

20. Tapia, J.E., Perez, C.A.: Gender classification based on fusion of different spatial scale features selected by mutual information from histogram of lbp, intensity and shape. IEEE Transactions on Information Forensics and Security 8(3), 488-499 (2013)

21. Wolf, L., Hassner, T., Taigman, Y.: Descriptor based methods on the wild. In: Faces in Real-Life Images Workshop in ECCV (2008) 